\title{
Investigação sobre o comportamento de uma Pessoa com Deficiência para a Construção de um Modelo de Arquitetura Mental Digitalizada que a reproduza como um Aprendiz
}

\author{
Rubens dos Santos Guimarães ${ }^{1}$, Válter Strafacci Júnior ${ }^{2}$, Paulo Marcelo Tasinaffo ${ }^{3}$ \\ ${ }^{1}$ Instituto Tecnológico de Aeronáutica (ITA), Praça Marechal Eduardo Gomes, 50, FCMF, \\ 12.228-900, São José dos Campos, SP, Brasil. \\ rubens.guimaraes@uol.com.br, strafaci@ita.br, tasinafo@ita.br
}

\begin{abstract}
Despite of having aggregate concepts from Psychology and Neuroscience, the informatics has shown less powerful than what was supposed to be at the beginning of the treatment on people with disabilities. The need to shape the cognitive human process play an extremely important role that justifies all the efforts concentrated on this direction. According to it, this job presents an investigation on people with disabilities behavior, applying it on a development of a Digital Mental Architecture model, which will provide a gain on the pedagogical structure on courses and follow up/ evaluation in real time for the process of teaching-learning of these people.
\end{abstract}

Resumo. A Informática, ainda que tenha agregado apropriadamente conceitos extraídos de outros Domínios de Conhecimento, como a Psicologia e a Neurociência, tem se mostrado menos poderosa e eficiente do que em princípio se esperava, e também menos realizadora do que se propunha principalmente no que diz respeito ao trato com Pessoas com Deficiência. A necessidade premente de modelar os processos cognitivos humanos desempenha papel de extrema importância que justifica a grande concentração de esforços nessa direção. Segundo este enfoque, este trabalho apresenta uma investigação do comportamento de Pessoas com Deficiência, aplicando-a na construção de um Modelo de Arquitetura Mental Digitalizada, a qual propiciará um ganho na estruturação pedagógica de cursos e acompanhamento/avaliação para o processo de ensino-aprendizagem destas pessoas.

\section{Introdução}

Com o crescimento das possibilidades do mundo virtual sustentado por hardware e software cada vez mais sofisticados, ampliaram-se os horizontes para a inclusão socioeconômica e cultural das Pessoas com Deficiência, com abrangência suficiente para atender as determinações da Convenção sobre os Direitos das Pessoas com Deficiência, que define que tais pessoas têm o direito a desenvolver suas capacidades e habilidades ao máximo (ONU, 2006).

Muito embora, os educadores busquem a melhoria do processo ensinoaprendizagem por meio de recursos pedagógicos, em muito deixam a desejar quando uma pessoa com deficiência se relaciona com tais recursos.

Tal distanciamento, pode ser atribuído ao desconhecimento sobre como se comporta uma pessoa com deficiência diante de seus desafios e capacidades de percepção, visto que o elo entre o factível e o desconhecido ainda não fora apropriadamente pesquisado e implementado.

Segundo (CLANCEY, 1995), não se pode reproduzir os padrões de comportamento humano sem reproduzir o mecanismo que produz o comportamento humano. Para tanto, a construção de um Modelo de Arquitetura Mental Digitalizada, proposta neste trabalho visando apoiar o processo de ensino-aprendizagem de pessoas com deficiência, sustenta-se 
no estudo do comportamento humano diante das incertezas e ambiguidades das condições de contorno que revestem o aprendiz.

\section{Investigação}

Todo o aprendiz, como ser humano, é suscetível de apresentar determinadas deficiências ou dificuldades temporárias ou permanentes, não significando desta maneira, restrições para um melhor ou pior desempenho de uma atividade socioeconômica ou cultural.

De acordo com a teoria de aprendizagem de Piaget (PIAGET et al, 1985), o desenvolvimento cognitivo de aprendizes encontra-se associado a quatro fatores: maturação biológica; experiência com o ambiente físico; experiência com o ambiente social; e equilíbrio. O equilíbrio é uma tentativa de trazer um estado de estabilidade entre os três primeiros fatores e a realidade associada com o ambiente externo. Durante cada fase do desenvolvimento, as pessoas se conduzem por meio de determinadas lógicas peculiares, estruturas mentais que lhes facultam adequadamente, fazer sentido do mundo. Isto ocorre mais fortemente ainda, em pessoas com deficiência, devido as suas restrições sensoriais e cognitivas.

Neste contexto de possibilidades, estes sintomas sinalizam um processo de aprendizagem para o aprendiz. Quando é desafiado a questionar, quando se perturba e necessita pensar para expressar suas dúvidas, passando a desenvolver a competência para formular e questionar problemas (SCHLEMMER, 2005).

A forma proposta neste trabalho, para desempenhar esta função "especular" será a de construir um Modelo de Arquitetura Mental Digitalizada - AMD, baseado numa estruturação concatenada da modelagem dos cinco sentidos, suas respectivas transitoriedades e relacionamentos associados a seus respectivos atributos (STRAFACCI, 2006).

A Figura 1 apresentada a seguir, representa o Diagrama de Classe (BOOCH;RUMBAUGH;JACOBSON, 2006) de uma AMD.

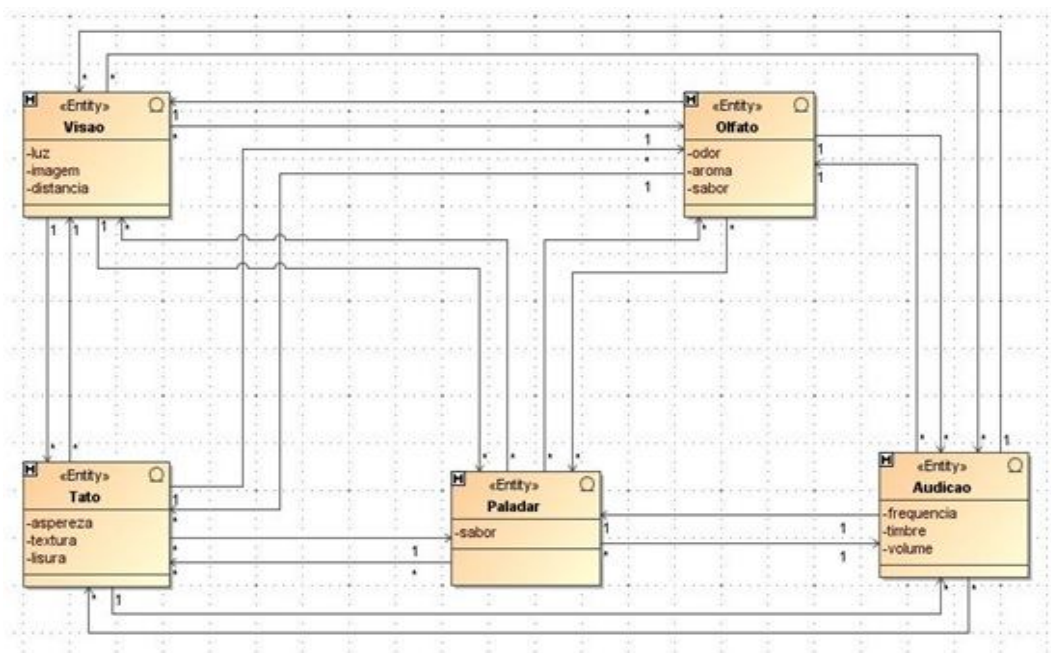

Figura 1. Diagrama de Classes de uma AMD

Ao modelo da AMD se incorporará os conhecimentos de Lógica Fuzzy (PEDRYCZ; GOMIDE, 2007), visando permitir o livre trânsito daquilo que o computador depreenderá sobre o estado psicometabólico do aprendiz. Dessa forma, poderá se estabelecer o nível de suficiência de transmissão de um conhecimento ou conteúdo, bem como a importância da consolidação do aprendizado e sua aplicação no cotidiano. 
Este modelo computacional originou o Sistema AMD, que aplicado à educação, propiciará obter-se o máximo de cada um dos cinco sentidos em função de seus respectivos mínimos de habilidade, para fornecer uma resposta mais apropriada para o relacionamento "Máquina-Homem" no sentido de apoiar o processo ensino-aprendizagem.

\section{Fundamentação Teórica para a Sustentação da AMD}

As abordagens adotadas nesta Pesquisa, quando associadas ao desenvolvimento de um Modelo Pedagógico, propiciarão uma melhor interpretação das necessidades e suficiências de um Programa de Ensino, e ainda a possibilidade de uma análise instantânea das ocorrências ao longo do seu desenvolvimento, previstas ou não numa programação inicial, as quais podem melhorar ou piorar o desempenho global esperado.

Para esse Trabalho será adotado a divisão das fases de um Programa de Ensino, segundo a conceituação apresentada a seguir.

As fases de um Programa de Ensino, dividem-se nas quatro seguintes: Estruturação Pedagógica, Distribuição Temporal, Aplicação e Consolidação.

Inicialmente, concebe-se um Programa de Ensino, baseado na constatação de uma necessidade e utilidade para uma pessoa com deficiência. Isto gera um conjunto de objetivos e metas da idealização.

Em seguida, a Distribuição Temporal transforma os resultados da Fase de Estruturação Pedagógica em planos, que suportam o Planejamento Executivo das atividades, relacionadas com os métodos e processos escolhidos. Uma vez planificado, passa-se à Fase de Aplicação da AMD, na qual também se controlam todas as etapas das atividades.

Na Consolidação, elabora-se todo o Material e a sua entrega ao Corpo Pedagógico.

O desenvolvimento, ao longo das Fases do Programa de Ensino, gera um conjunto de Informações, as quais devem ser tratadas de maneira apropriada, visando solucionar situações conflito, tanto os previsíveis quanto as imprevisíveis.

Esta distribuição de fases, que atende a elaboração de um programa de ensino será transferida para o emprego da AMD para um determinado estudante, sendo seu acompanhamento efetuado no dia a dia e representado por uma curva monotônica ascendente, como forma de avaliar seu desempenho num determinado seguimento do conhecimento.

Todo processo desenvolve-se por meio da manipulação de informações. As mesmas podem ser geradas, interna ou externamente, ao processo no qual estejam inseridas. Este conjunto de informações é processado como "entrada" e geram informações de "saída" transformadas por um processo.

Num processo qualquer de desenvolvimento ou aplicação, a matéria-prima básica manipulada são as Informações, capazes de indicar e de alterar o comportamento de diversos segmentos do projeto de uma ação qualquer, no caso desta pesquisa, a Pedagógica.

Para fins desta sustentação, Informação refere-se a toda e qualquer comunicação processada de maneira formal ou informal, relacionada com um fato ou ocorrência, que possa influenciar, positiva ou negativamente, no desenvolvimento de um Projeto (MARTIN, 1984).

Em função da percepção da importância no tratamento das informações, a AMD proposta estruturar-se-á de maneira a tratar o diagrama de classes apresentado na Figura 1 para construir as regras Fuzzy, cujos resultados alimentarão um conjunto de redes neurais, criando uma estrutura de Neuro Fuzzy. 
Os valores relevantes a serem apontados por essa estrutura serão os seguintes:

- $\quad \mathrm{k}$ - indicativo da condição específica de uma Pessoa com Deficiência - PD;

- fator de impacto - a resposta de uma PD e do sistema diante de uma determinada ocorrência;

- $\quad$ esforço - valor indicativo da energia despendida por uma PD para equilibrar os efeitos de uma Informação;

- consolidação - valor indicativo do aprendizado de um conteúdo programático, relacionado com a percepção de uma informação;

- fase - época ou período do programa em que o aprendiz se encontra;

- avaliação/teste - indicativo da validade dos processos de avaliação como verificadores do aprendizado;

- avanço didático - indicativo do ganho de conhecimento num determinado domínio do conhecimento aplicado no cotidiano;

- inclusão social - indicativo da qualidade do relacionamento da PD inserida num contexto pedagógico educacional frente ao ambiente;

- verificação final - indicativo do Valor de Estado da aplicação de um Programa de Ensino em um conjunto, unitário ou plural de PD;

- zelo comportamental - aplicação do aprendiz na condução dos estudos, frequência, dentre outros; e

- aplicação do aprendizado - a utilização do transmitido no cotidiano: validação do conteúdo programático.

O resultado da análise destes elementos propiciará o acompanhamento e a consolidação do aprendizado de pessoas com deficiência.

\section{A AMD: Estruturação e Aplicação}

O Sistema AMD integra-se ao contexto de sistemas computacionais como fundamento central da ciência cognitiva moderna (CHALMERS, 1993).

A Figura 2, apresenta a principal interface de entrada de dados do Sistema.

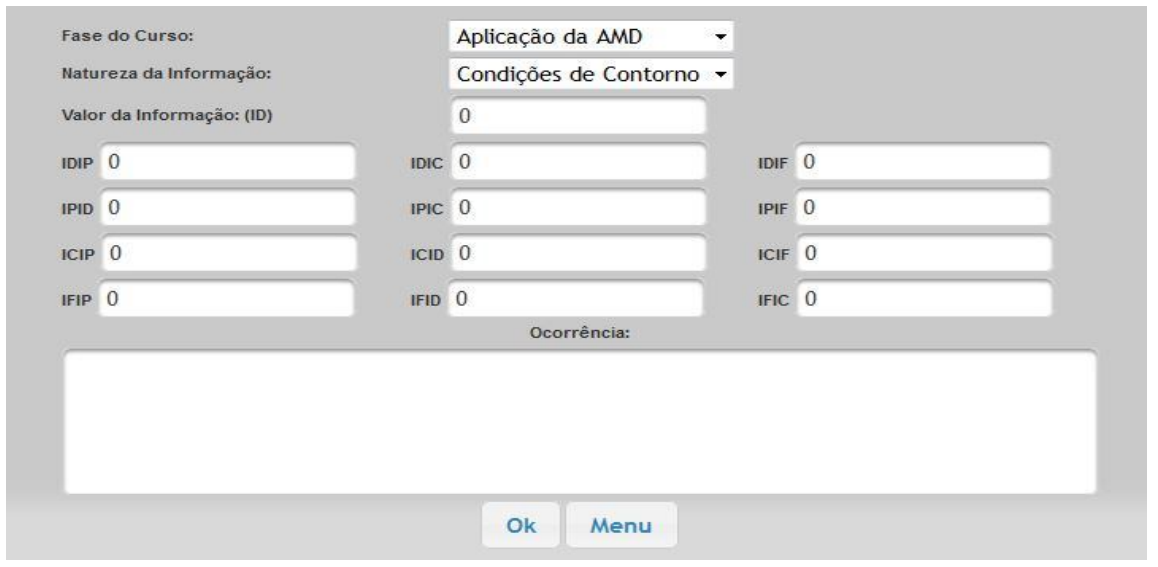

Figura 2. Entrada de dados para processamento da AMD

Este sistema foi desenvolvido em Java no ambiente NetBeans (ORACLE, 2012) e sua interface gráfica com o framework JSF (GEARY;HORSTMANN, 2010). Ele utiliza o MySQL (WELLING, 2005) como gerenciador de banco de dados.

Trata-se de uma aplicação WEB, onde apresenta-se um formulário principal com 
argumentos da Lógica Fuzzy suportando todas as regras de inferência da dedução natural.

O processo de análise de informações, que permite avaliar o desenvolvimento das habilidades de uma pessoa com deficiência, ocorre a partir da seguinte entrada de dados: Fases do Curso (ou Projeto Pedagógico); Natureza da Informação; e Valores da Informação.

As Fases do Curso são divididas em: Estruturação Pedagógica, Distribuição Temporal, Aplicação da AMD e Consolidação.

Com relação à Natureza, as Informações classificam-se em: Condições de Contorno, Capacidade Física, Dúvida de Conteúdo e Condição Psicoemocional.

Essa classificação determina onde o Processo de Análise iniciar-se-á, baseado na Natureza da Informação comunicada.

Essa percepção, transferida para o processo, posicionará as máquinas de Lógica Fuzzy, que tratarão as Informações e suas respectivas influências.

A própria identificação da Natureza de uma Informação pode ser considerada dentro de uma ambiguidade, pois depende da percepção particular de cada Educador. Nessas oportunidades, a comunicação interfuncional pode minimizar a ambiguidade pelo compartilhamento da responsabilidade na decisão sobre a classificação.

Cada Informação deverá vir acompanhada de um Fator de Influência, sobre cada uma das outras, e um Fator de Influencia Recíproca.

Considera-se Fator de Influência - FI a pressão que a constatação de uma informação exerce sobre as demais, numa relação biunívoca. Essa relação cria um Fator de Influencia Recíproca - FI $\mathbf{r}_{\mathbf{r}}$, o qual representa a resposta da informação influenciada, a àquela que provocou a comunicação da informação.

De acordo com um exemplo de entrada de dados apresentado na Figura 2, para quaisquer Fases do Curso informada, ao selecionar a Natureza da Informação, o sistema solicita automaticamente determinados Fatores de Influência.

A indicação dos Fatores de Influência conforme a Natureza da Informação selecionada segue a seguinte notação:

ICIP - Influência das Informações de Condições de Contorno sobre as Informações Psicoemocionais;

ICID - Influência das Informações de Condições de Contorno sobre as Informações de Dúvidas de Conteúdo;

ICIF - Influência das Informações de Condições de Contorno sobre as Informações de Capacidade Física;

IPID - Influência das Informações Psicoemocionais sobre as Informações de Dúvidas de Conteúdo;

IPIC - Influência das Informações Psicoemocionais sobre as Informações de Condições de Contorno;

IPIF - Influência das Informações Psicoemocionais sobre as Informações de Capacidade Física;

IDIP - Influência das Informações de Dúvidas de Conteúdo sobre as Informações Psicoemocionais;

IDIC - Influência das Informações de Dúvidas de Conteúdo sobre as Informações de Condições de Contorno; 
IDIF - Influência das Informações de Dúvidas de Conteúdo sobre as Informações de Capacidade Física;

IFIP - Influência das Informações de Capacidade Física sobre as Informações Psicoemocionais;

IFID - Influência das Informações de Capacidade Física sobre as Informações de Dúvidas de Conteúdo; e

IFIC - Influência das Informações de Capacidade Física sobre as Informações de Condições de Contorno.

Os Fatores de Influência - FI podem então assumir valores, desde altamente negativos, passando por neutro, até altamente positivos, conforme a informação do usuário.

Essa faixa deverá variar desde o valor de $-1,00$ (um negativo), para o primeiro caso, passando por "zero", até $+1,00$ (um positivo), no último caso. Esses valores poderão ser informados para o processo com até duas casas decimais.

Definidos os valores dos FI, avalia-se a condição da Informação comunicada que pode ser considerada "muito ruim", "ruim", "correta", "boa", ou "muito boa".

A Tabela 1 apresenta os valores atribuídos a cada uma das faixas e suas respectivas pertinências. Essas últimas têm a função de definir os intervalos que geram os planos criados pela aplicação para o tratamento das Informações pelas máquinas de Lógica Fuzzy.

\section{Tabela 1. Condição, Valor e Pertinência das Informações}

\begin{tabular}{|l|c|c|}
\hline Condição & $\begin{array}{c}\text { Faixa de } \\
\text { Valor }\end{array}$ & Pertinência \\
\hline Muito ruim & 0 a 0,245 & 1,00 a 0,5 \\
\hline Ruim & 0,245 a 0,49 & 0,5 a 0 \\
\hline Correta & $\begin{array}{l}0,49-0,5 \text { e } \\
0,51\end{array}$ & $0-1,00$ e 0 \\
\hline Boa & 0,51 a 0,755 & 0 a 0,5 \\
\hline Muito boa & 0,755 a 1,00 & 0,5 a 1,00 \\
\hline
\end{tabular}

Os Fatores de Influência variando de $-1,00$ (menos um) até $+1,00$ (mais um), passando pelo "zero", representam respectivamente uma variação negativa, neutra e positiva. As pertinências são respectivamente 1,00 nos limites extremos e "zero" com valor "neutro". Quando "neutra", a pertinência assume o valor 1,00.

A coleta dos dados, fornecidos ao sistema pelos educadores (Professores, Orientadores Pedagógicos, entre outros) e supervisionados pelos Profissionais de Atendimento Educacional Especializado - AEE, são obtidos por meio dos fatores que caracterizam o perfil da capacidade cognitiva dos estudantes, tais como: de relacionamento, de vocação, de degradação funcional, entre outros.

A partir dos valores informados, montam-se as máquinas de Lógica Fuzzy, das quais extraem-se os resultados da análise da comunicação de uma Informação, de maneira integrada, relacionando-se todas as Naturezas e suas interdependências. Esses resultados são utilizados no cálculo do Valor de Estados.

As máquinas recebem os dados, "fuzificam", "defuzificam”, e apresentam o resultado referente a cada uma das Naturezas de Informação, baseadas numa base de conhecimento. 
Completada essa fase, repassam-se as Informações classificadas para o processamento de análise e interpretação, sobre o que elas representam para o Curso ou Projeto Pedagógico em questão, segundo uma visão global da situação.

Esses resultados gerados pelo sistema, propiciarão subsídios para uma melhor abordagem dos conteúdos apresentados aos estudantes com deficiência, sinalizando para ampliação das possibilidades de inclusão dessas pessoas.

Como forma de aplicar o modelo proposto, criou-se uma instância num domínio de conhecimento específico.

\section{Estudo de Caso}

Este trabalho propõe um Estudo de Caso sobre "Desenvolvimento de Habilidades para o Ensino de Pessoas com Deficiência”. Um protótipo será experimentado em algumas instâncias, no domínio de conhecimento da Física.

Foram construídas regras de Lógica Fuzzy responsáveis pelo tratamento das ambiguidades e incertezas decorrentes de toda e qualquer informação externa percebida por um aprendiz com Deficiência, que podem de uma ou de outra forma, alterar sua conduta diante do estudo de um determinado conteúdo programático.

As respostas da Lógica Fuzzy, responsáveis pela análise das influências diretas e recíprocas entre cada natureza de informação, alimentarão as Redes Neurais Artificiais RNA responsáveis pela construção da função de transferência do estado do estudante em relação ao seu processo de aprendizagem.

Para cada aprendiz inserido no sistema, será atribuído um valor "k" representativo de suas condições iniciais, calculado em função de uma série de fatores que caracterizarão seus respectivos perfis de capacidade cognitiva, de relacionamento, de vocação e degradação funcional.

Esse fator será monitorado ao longo da transmissão de um conhecimento específico. Conforme a avaliação das naturezas das informações percebidas ao longo de um período e seus respectivos impactos sobre um aprendiz, o Educador poderá estabelecer alterações ou adequações em sua conduta, bem como no seu critério de avaliação.

Para cada aprendiz será atribuída uma curva de avaliação e controle, distribuída em 4 fases (Estruturação Pedagógica, Distribuição Temporal, Aplicação da AMD e Consolidação), que cumulativamente agregará um Valor de Estado em função de cada uma das fases, até o valor limite final estabelecido logo do início de um determinado período de aprendizagem, tratado como Valor de Estado Final, o qual se pretende atingir e representa a potencialidade esperada do aprendiz.

\section{Experimentos e Análise de Resultados}

Esta pesquisa propõe-se a propiciar avaliações de experimentos realizados, por meio de um protótipo aplicado em um Estudo de Caso.

O experimento foi realizado no Colégio Estadual Barão de Aiuruoca, em Barra Mansa, por um dos Professores de Física da Instituição.

Foram assistidos um total de 5 alunos com deficiência. Deste grupo, um aluno possui Síndrome de Down, dois são autistas nível leve e os outros dois são surdos. Nesse experimento realizado, o total dimensionado de alunos, representou o tamanho de uma amostra, que segundo (MENDENHALL, 1994), é suficiente para obtenção de resultados satisfatórios e significativos.

A aplicação da AMD para os alunos assistidos, sinalizou para a utilização de algumas estratégias pedagógicas necessárias para um melhor rendimento da aprendizagem. 
Adotou-se a utilização de vídeo-aulas como ferramenta de apoio a aprendizagem (GUIMARÃES, 2008), pois verificou-se a necessidade da exploração do potencial visual dos alunos .

Em determinados momentos das aulas, os conteúdos apresentados enfatizaram itens de maior relevância para os deficientes, conforme a avaliação da capacidade cognitiva desses alunos, tornando a apresentação mais flexível às diferenças.

Utilizou-se uma avaliação diferenciada para os alunos deficientes, aplicou-se neste experimento específico um objeto de avaliação (GUIMARÃES, 2011), tornando possível e viável o tratamento das contradições (DA COSTA, 2007), possibilitando-se atingir a potencialidade esperada do aprendiz.

Para a aplicação da análise de resultados por testes de hipótese (HOWELL, 2002), referente ao desenvolvimento de Habilidades para o Ensino da Física para pessoas com deficiência, analisou-se o desenvolvimento dos alunos em dois períodos, considerando a aplicação da AMD no segundo. As médias atribuídas aos alunos após cada período, são mostradas na Tabela 2, a seguir.

Tabela 2. Notas referentes a avaliação dos alunos

\begin{tabular}{|l|c|c|}
\hline Aluno & Médias ${ }^{\circ}$ período & Médias 2 ${ }^{\circ}$ período \\
\hline Aluno 1 - Turma 1007 (Síndrome de & 75 & 80 \\
\hline Aluno 2 - Turma 1003 (Autista) & 70 & 72 \\
\hline Aluno 3 - Turma 1004 (Autista) & 60 & 65 \\
\hline Aluno 4 - Turma 1001 (Surda) & 72 & 78 \\
\hline Aluno 5 - Turma 1001(Surda) & 78 & 85 \\
\hline
\end{tabular}

Note-se nesta tabela, que os resultados auferidos pelos alunos no $2^{\circ}$ período, após a aplicação da AMD, são superiores aos do $1^{\circ}$ período, que muito embora de obvia constatação, deve ser ressaltado.

A análise dos resultados, obtida por meio de testes de hipótese (HOWELL, 2002), deixam claro que a faculdade cognitiva está intrinsecamente relacionada com uma necessidade, e que a fixação do aprendizado depende da forma e conduta pelas quais se apresenta o suficiente para a sua compreensão.

\section{Conclusão}

O objetivo principal desta pesquisa foi investigar, conceber e implementar um Modelo Conceitual para uma Arquitetura Mental Digitalizada - AMD para apoiar o processo de ensino-aprendizagem de pessoas com deficiência, sustentado em sua cultura e experiência de vida.

Para atingir a este objetivo, num Estudo de Caso sobre "Desenvolvimento de Habilidades para o Ensino de Pessoas com Deficiência", um protótipo aplicado ao domínio de conhecimento de Física foi construído e implementado em experimentos.

Os resultados dos experimentos mostraram que o protótipo atendeu satisfatoriamente as expectativas. 
Da percepção sobre o comportamento de pessoas com deficiência, geralmente circunspectas em seus mundos limitados pelas próprias barreiras impostas pela sociedade, será possível concatenar, decidir e optar pela construção de uma Arquitetura Mental Digitalizada capaz de representar a Interface Máquina-Homem segundo as possibilidades da transitoriedade das relevâncias das informações percebidas pelos cinco sentidos e decidir sobre um nível de suficiência para a apresentação de um conteúdo programático de estudo.

Dessa forma, torna-se possível transferir para o sistema o máximo que uma pessoa com deficiência se apropria do mínimo que seus sensores são capazes de perceber e interpretar.

Os autores desta pesquisa acreditam que a inclusão social de pessoas com necessidades especiais, por meio de um protótipo como o Sistema AMD, devidamente aperfeiçoado, não só pode como deve propiciar, através do uso de tecnologias apropriadas, a redução de desigualdades e promover maior bem estar à sociedade.

\section{Referências}

BOOCH, Grady; RUMBAUGH, James, JACOBSON, Ivar. Unified Modeling Language: UML (Guia do Usuário). Campus: São Paulo, 2006.

CHALMERS, D.J. A Computational Foundation for the Study of Cognition. 1993. Disponível em: < http://consc.net/papers/computation.html>. Acesso em: 24 Jul. 2013.

CLANCEY, W.J. A boy scout, Toto, and a bird: How situated cognition is different from situated robotics. Em: Steels, L. e Brooks, R. (eds.) The "artificial life" route to "artificial intelligence": building situated embodied agents. Hillsdale, NJ: Lawrence Erlbaum Associates. 1995.

DA COSTA, N.; KRAUSE, D. ; BUENO, O. Paraconsistent Logics and Paraconsistency. In: Gabbay, D.; Thagard, P.; Woods, J. (eds) Handbook of the Philosophy of Science. Volume 5: Philosophy of Logic, 2007.

GEARY D., HORSTMANN C. Core JavaServer Faces, 3. ed. Boston: Pearson Education, 2010.

GUIMARÃES, R. “ACEAS: Um Ambiente Computadorizado de Ensino e Aprendizagem para Surdos". Anais do SBIE 2008, Fortaleza, 2008.

GUIMARÃES, R. "Uma Ferramenta de Lógica na Contradição da Avaliação de EnsinoAprendizado". Anais do WIE 2011, Aracaju, 2011.

HOWELL, D., Statistical methods for psychology (5th ed.). Pacific Grove, CA: Duxbury/Thomson Learning. 2002.

MARTIN, J. An Information Systems Manifesto. Prentice Hall, Inc., Englewood Cliffs, New Jersey, USA, 1984.

MENDENHALL, Willian et all, Introduction to Probability and Statistics, Wadsworth Pub Co., $9^{\text {Th }}$ Edition -1994.

ONU 2006, Convenção sobre os Direitos das Pessoas com Deficiência, adotada em 13 de dezembro de 2006.

ORACLE Inc. Welcome to NetBeans. 2012. Disponível em <http://netbeans.org>

PEDRYCZ, W.; GOMIDE, F. Fuzzy systems engineering: toward human-centric computing. New York: Wiley-IEEE Press, 2007.

PIAGET, J., BROWN, T., and THAMPY, K. Equilibration of Cognitive Structures: The Central Problem of Intellectual Development. University of Chicago Press. 1985.

SCHLEMMER, E. "Metodologias para educação a distância no contexto da formação de comunidades virtuais de aprendizagem", Ambientes Virtuais de Aprendizagem, Artmed, $1^{\text {a }}$ ed., Porto Alegre. 2005.

STRAFACCI, V., Gerência Perceptiva, a Expansão da Produtividade Cerebral, Stratégia Propaganda, 2006.

WELLING, Luke. PHP e MySQL desenvolvimento web, Elsevier, 2005. 